\title{
Editing Templates: A User Interface Generation Tool
}

Dan R. Olsen Jr.

dan_olsen@byu.edu

Follow this and additional works at: https://scholarsarchive.byu.edu/facpub

Part of the Electrical and Computer Engineering Commons

\section{Original Publication Citation}

Olsen, D. R. "Editing Templates: A User Interface Generation Tool." Computer Graphics and Applications, IEEE 6.11 (1986): 4-5

\section{BYU ScholarsArchive Citation}

Olsen, Dan R. Jr., "Editing Templates: A User Interface Generation Tool" (1986). Faculty Publications. 750. https://scholarsarchive.byu.edu/facpub/750 accepted for inclusion in Faculty Publications by an authorized administrator of BYU ScholarsArchive. For more information, please contact ellen_amatangelo@byu.edu. 


\section{Editing Templates: \\ A User Interface Generation Tool}

Dan R. Olsen, Jr.

Brigham Young University

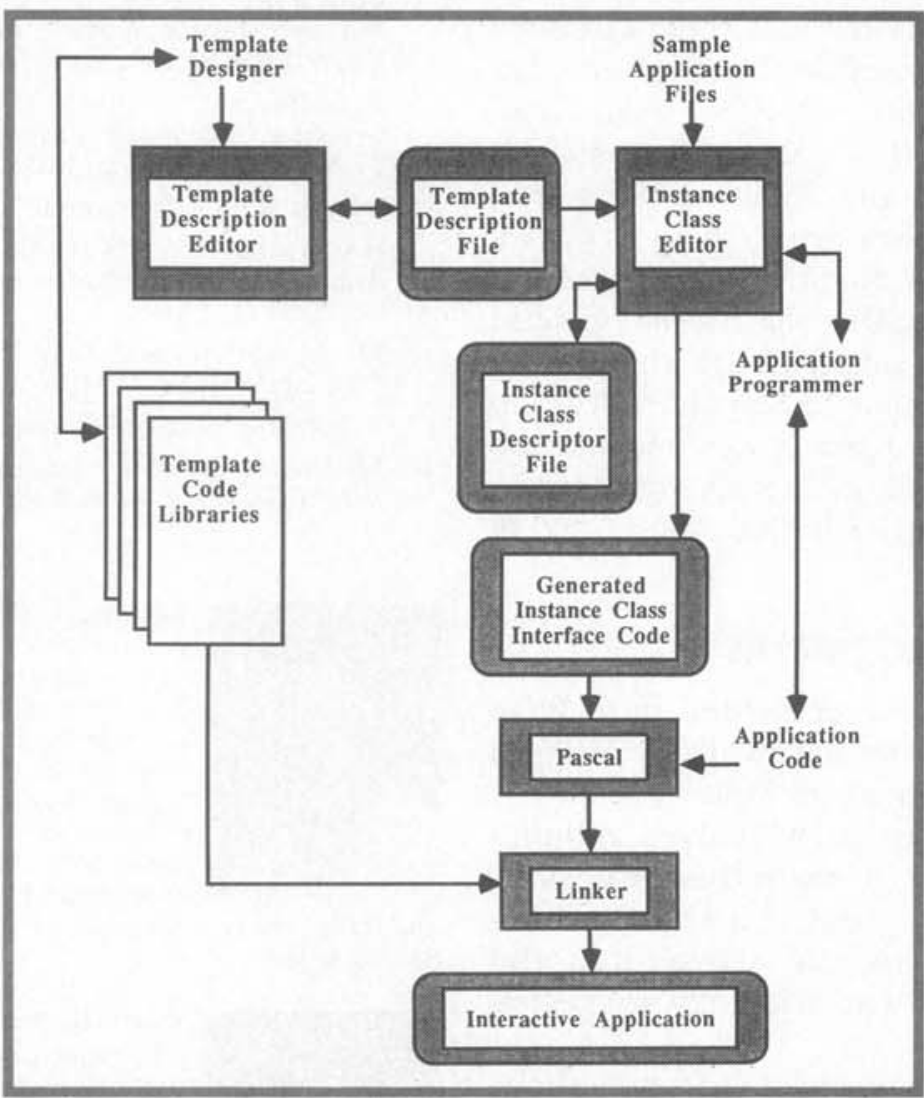

This article discusses a basic architecture for a user interface management system and the problem of updating a display in response to interactive commands. The basic architecture is then extended to include basic editing and browsing processes on arbitrary data structures. Editing templates are presented as a technique embodying the entire manipulation process for a particular data structure/data display combination. In conjunction with the user interface management system, such templates are able to automatically provide a majority of the code required in an interactive application.
$\mathbf{T}$ he concept of a user interface management system (UIMS) has become well known in the graphics community. These systems have been developed to overcome the high cost of implementing interactive graphics programs with quality human-computer interfaces. Most systems have concentrated on the problem of input dialogue

An earlier version of this article appeared in Proc. Graphics Interface 86. management. ${ }^{2-5}$ Having developed three such systems, ${ }^{6-9}$ we at the Brigham Young University Computer Science Laboratory have become concerned with the problem of display in an interactive program. In implementing interactive programs, we have found that the input dialogue can be programmed in a matter of hours or days, using our tools, but the code to update the display after each modification to the application data structure takes 
months to implement. We approached this display update problem with an intelligent display processor. Our original architecture for an interactive program is shown in Figure 1.

In this architecture the input events are parsed according to the dialogue description and, on the basis of the input, one or more of the application command procedures are invoked. These application command procedures are responsible for updating some application data structure. The display processor creates a graphical presentation of the application data according to the specified format. Here I will only touch upon the wide range of possible formats for displaying the data.

This architecture is somewhat similar to the one proposed at the Seeheim workshop on user interface management..$^{10}$ The key issue in this article is how the graphical image should be updated whenever the application data is changed. The obvious solution is simply to redraw the entire image, yet this is an extremely poor solution if we desire reasonable response time. After some experience with the model shown in Figure 1, we determined that a closer relationship between the data-editing commands and the display update function is essential. A more acceptable system architecture is shown in Figure 2.

In this architecture a large portion of the interactive dialogue is viewed as a process of browsing and/or editing the application data structure. Therefore, a description of the application data is taken together with a selection of browsing and editing methods to generate the dialogue definition, display update procedures, and application command procedures for the selected operations. Note that this procedure does not generate the entire interactive program but simply the editing portion, which is the most display-update intensive. Any analysis, file management, or help facilities can be added. Our experience shows that the editing and browsing operations dominate an interactive program.

This article will review a data management facility called STUF (STrUctured Files) to define the data structures that might be represented in this UIMS model. A short description of MIKE (Menu Interaction Kontrol Environment), our input dialogue system, will follow. With the stage set for the editing environment, editing templates that provide the desired screen update facilities will be described.

\section{STUF}

The STUF package was developed as a data model specifically for interactive applications. We wanted to create a data model that would behave equally well in main memory or on secondary storage. We also imposed the requirement that STUF data must

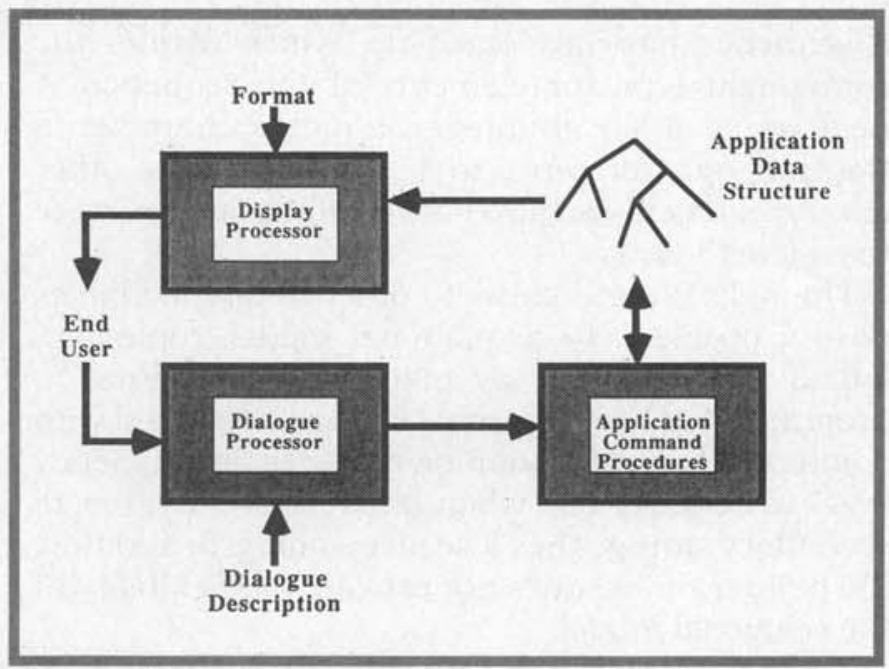

Figure 1. A UIMS display architecture.

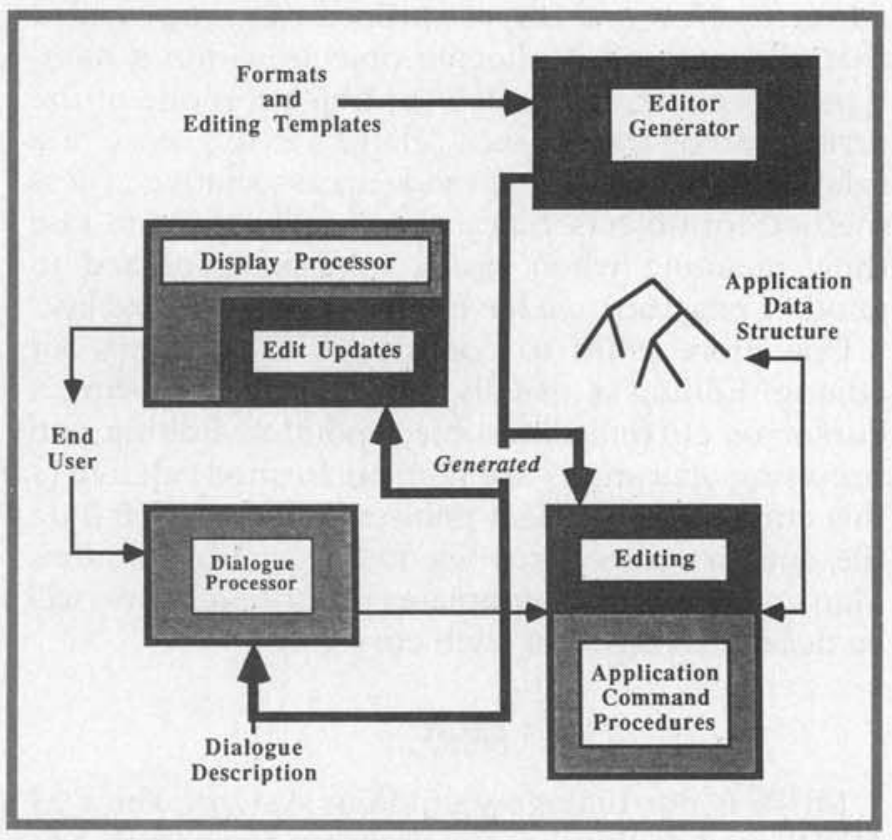

Figure 2. Editing templates architecture.

be accessible either relationally (as in a relational database) or as a linked structure (as with pointers in Pascal), because, despite their power, relational data models are often too inefficient for practical graphics use.

Each STUF file has a specific filetype defined by a set of datatypes. A datatype is a record, a union, or a variable-length sequence, and consists of a list of fields, each of which has a fieldtype. A record has all of the fields in the list. A union has one of the fields in the list (as determined by a tag field at runtime) and is similar in capability to a Pascal variant record. 
A sequence has only one field, which defines the component type for elements of the sequence. A fieldtype is either an integer, a real, a character, a Boolean, or a reference to an object of some other datatype. A field can also have a dimension to create fixed-length arrays.

The links or references to other datatype objects pose a problem. In a relational model, tuples are linked to each other by matching key values. In programming languages this linkage is handled with pointers. The pointer solution provides the efficiency that we require, but when pointers are written to secondary storage they lose all meaning. In addition, the pointer model does not provide the flexibility of the relational model.

The STUF solution is to create, for each datatype in the file, a variable-length array of objects. A reference to an object is then stored as an index into this array. New and Dispose procedures are provided that allocate and deallocate objects within a datatype. Moreover, the undeleted objects in one of the arrays can be treated as a relation, in the sense of a relational database, to provide an associative access method for objects. Since object indices do not lose their meaning when saved to disk or passed to another program, we have our needed data facility.

One more point to consider is the cursors for editing. Editing is usually performed by moving a cursor or current data object pointer. Editing and browsing operations are then performed relative to this current data object pointer. With a STUF data file, such a cursor is represented as an object index. Many of the editing templates described below will be defined in terms of such cursors.

\section{MIKE}

MIKE is our dialogue-handling system. The way MIKE models the input dialogue is important: The basic interactive unit in MIKE is the command, simply a Pascal procedure or function. MIKE accepts as its initial dialogue description a set of such procedures and then generates a compiled interface to them.

All procedures are presented interactively in a menu, and the selected menu item becomes the current command. Having selected a command, MIKE then prompts with a menu of all functions whose result type is the same as that of the command's first parameter. These types can be from all types in the application program.

In addition to the functions in the menu, MIKE can supply primitive inputs for integer, real, string, function key, and point types. MIKE continues accepting inputs until a complete command expression has been parsed. The command expression syntax is very similar to Pascal procedure invocation syntax, except that no punctuation, such as commas and parentheses, is actually input. MIKE's interactive use of functions and procedures is similar to Smalltalk's interactive use of methods. ${ }^{11}$

A profile editor can improve such a primitive interface. The profile editor allows menus to be restructured, prompts and echoes changed, icons drawn, function buttons mapped to commands, and help texts written. It fleshes out and enhances the user's view of the interface but does not change the underlying model of command procedures and functions. The profile editor serves a role similar to that of Buxton's MenuLay ${ }^{12}$ in editing external presentations of dialogues.

This command model has proved to be much simpler than the state machine and grammar approaches that we have used. For our editing model of interaction, we can characterize all changes to the application data structure as Pascal procedures or functions. In other words, for each kind of change to be made to the data structure, we write a procedure that makes the change and performs any necessary display updates. The generator then informs MIKE of the procedure names and parameters, which make up the dialogue interface.

\section{Editing templates}

The editor generation concept is based on editing templates. An editing template is designed as a presentation of a general class of data structures. Linked lists, symbol tables, and trees are examples of such structure classes. Thus, an editing template consists of a set of routines to do the following:

1. Display application data from a STUF file using the model.

2. Provide data structure traversal commands for browsing through the data image displayed by the model.

3. Provide the editing commands for creating, deleting, and/or modifying the data presented using the model.

The commands provided must, in addition to performing their intended tasks, update the display to reflect the results of their tasks. The display update is very important here.

In addition to the services it provides, each template has a set of parameters for creating an instance of a template. An editing template can be thought of as a macro, except that the parameters may be lists and other data structures rather than simple text to be substituted.

In Smalltalk, an editing template would be defined as a class. The services that it provides would be methods of the class, and the parameters would be methods of the objects that the template manipu- 
lates. In Ada an editing template could usually be represented as a generic package..$^{13}$ Our work has been done in Pascal using a macro preprocessor, but the concepts are the same. Thus an editing template is characterized by the data structure that it represents.

\section{Linked-list editing template}

A linked list is a good first example of how editing templates function. Since an editing template is meant to be a generic capability, it must know many things about the linked list that it is to display. For example, it must know how to find the head of the list, what field is used as the link for the list, how to display one of the elements of the list, and how much display space to allocate to each element. This information is provided by the following set of parameters. The parameters are all preceded by a percent sign so that their text is easily recognized in the generated routines.

$\%$ ObjType-The datatype of the list elements to be displayed.

$\%$ Link-The field used to link elements of $\% O b j$ Type together.

$\% \mathrm{CurObj-The} \mathrm{tuple} \mathrm{index} \mathrm{variable} \mathrm{used} \mathrm{as} \mathrm{the}$ cursor in moving up and down the linked list. This is an index into \%ObjType's array.

$\%$ HeadType-The type of object where the head of the list is stored.

$\%$ HeadField-The field in \%HeadType that points to the head of the linked list being displayed. This field must reference datatype \%ObjType.

$\% \mathrm{CurHeadObj-The} \mathrm{tuple} \mathrm{index} \mathrm{variable} \mathrm{that}$ references the \%HeadType tuple containing the head of the current list being displayed.

$\% X E x t$ and \%YExt-The $\mathrm{X}$ and $\mathrm{Y}$ sizes of the screen space to be allocated to each displayed element.

$\%$ Window-The number of the window that the list is to be displayed in.

$\%$ ObjDisp (\%ObjIndex; $X, Y$ )-A routine to be called to have the list element referenced by $\% O b j$ Index displayed on the screen at location $(X, Y)$.

$\% O b j \operatorname{Del}(\% O b j I n d e x)-A$ routine that will clean up and delete the element referenced by $\% O b j$ Index.

\%EditSpace-The number of list element spaces to be left empty on the screen so that new elements can be inserted into the list without repainting the entire list.

The various interactive tasks that we want to perform on the display of a linked list include moving a cursor up and down the list (scrolling the list if necessary), inserting a new element in the list, and deleting the current element from the list. In addition to these primitive operations, we might want to select a current item from the list using some other criterion such as a name. To accomplish all these tasks, the template would provide the following command procedures directly to MIKE:

\%Window Up

\{move the list cursor up one element, scrolling if necessary

\%WindowDown

\{move the list cursor down one element, scrolling if necessary

\%WindowLeft and \%WindowRight

\{if, because of the size and shape of the window, the list is displayed in multiple columns, move the cursor left or right as the case may be and update \%CurObj appropriately

\%WindowPageUp and \%WindowPageDown

\{if the list is longer than will fit in the window, move the cursor backward and forward through the list one page at a time

$\%$ WindowDelete

\{delete the current object pointed at by $\%$ CurObj $\}$

In addition to these command procedures exposed to MIKE, the following additional service routines are generated:

\section{Restore\%Window}

\{refresh the window whenever the window itself changes or the value of \%CurHeadObj changes \}

$\%$ WindowUpdateCur

\{update the display of the current object due to some modification to it by some other command\}

\%WindowInsert (\%ObjIndex)

\{insert the specified object into the linked list immediately after \%CurObj and make it the current object, updating the display appropriately

$\%$ WindowChangeCursor (\%ObjIndex)

\{change \%CurObj to the value of \%ObjIndex and update the screen appropriately

Note that all of the names of the generated routines are parameterized by $\%$ Window to make them unique. Also, there is no insert command exposed directly to MIKE because of the variety of ways that an application may want to create and initialize elements of the list. After such an element is created by a command procedure, the \%WindowInsert service procedure can be called to handle the listinsertion and screen-update tasks properly.

The actual display of an element is left to an application-supplied procedure. Often we have had lists of lists to display. The procedure is handled with two windows, \%W1 and \%W2, for example. The main list is displayed by $\% W 1$ and can be browsed 


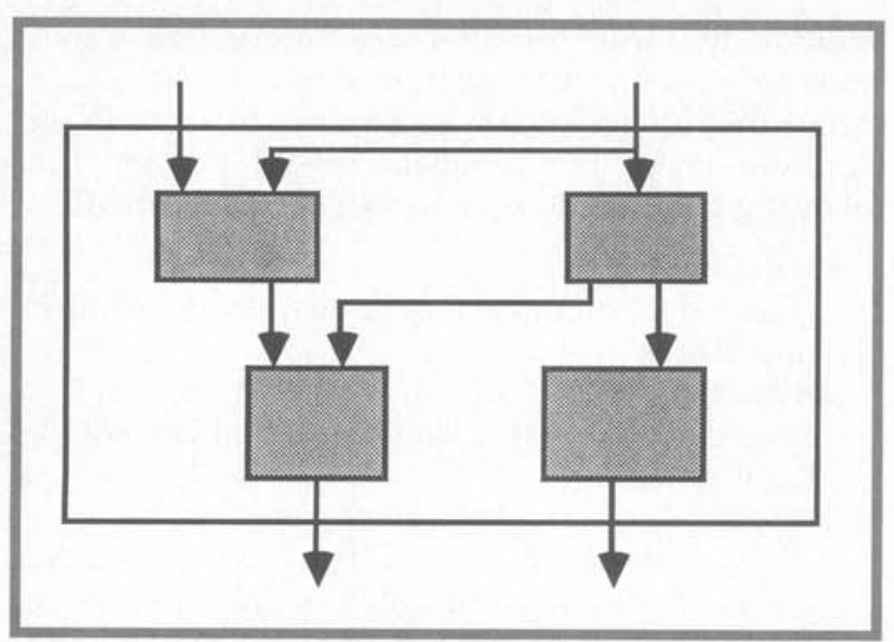

Figure 3. Schematic template.

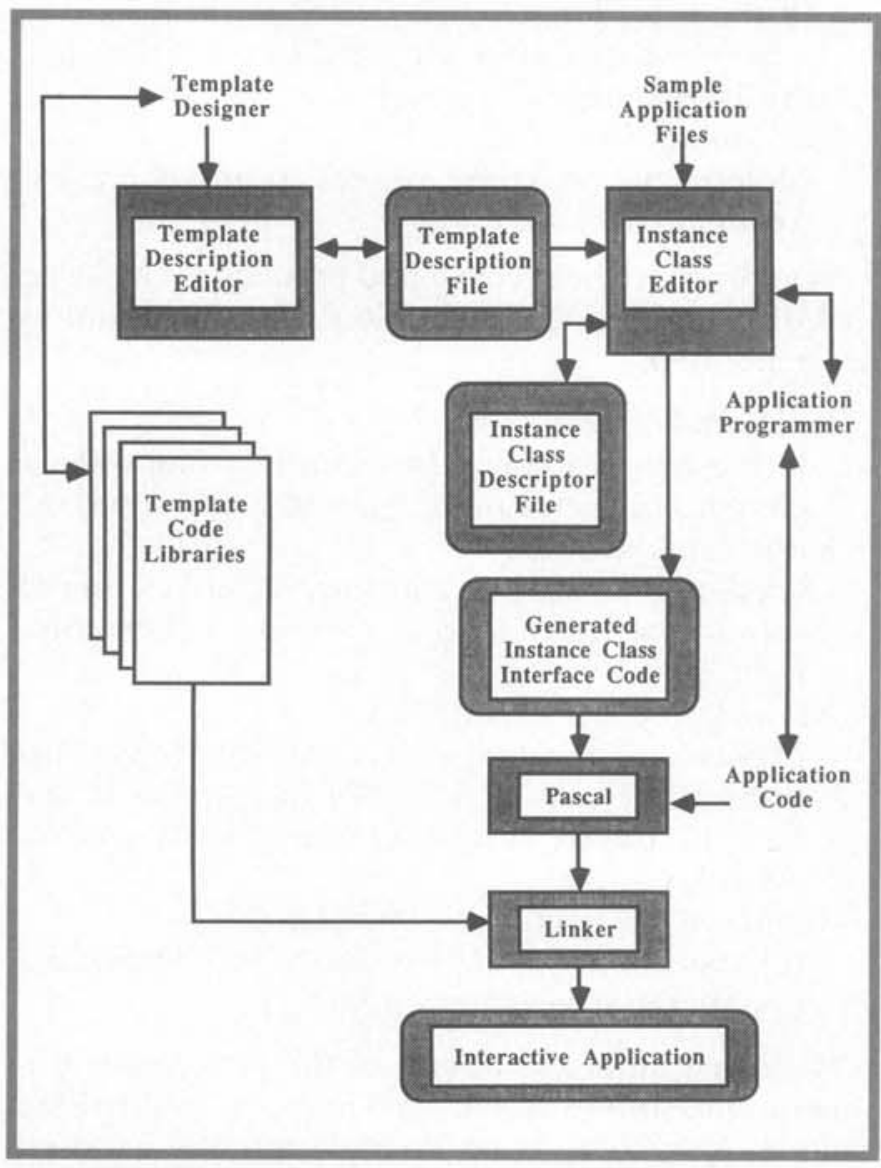

Figure 4. Detailed editing templates architecture.

and edited as shown above; $96 \mathrm{W1}$ 's $96 \mathrm{CurObj}$ cursor is also the 96 CurHeadObj for 962 so that any change of the cursor in $\% \mathrm{Wl}$ will cause an update of 96 W2. Combining the linked-list template with the various other templates implements a large number of data display and manipulation techniques very quickly.

\section{Other possibilities}

The set of services provided above is not necessarily complete nor is it the only possible approach. For example, the linked-list template could have a clipboard variable added as a parameter and then supply to MIKE the necessary commands to Cut, Paste, and Copy list segments to and from the window's clipboard. An additional feature might select an element from the list with a mouse rather than scroll through the list.

Other data-structuring mechanisms we have implemented include unordered and sorted associative lists. These lists are accessed by name or some other criterion. These two techniques view a given STUF datatype as a table of tuples for a relation and allow scrolling through and editing of such tables based on application-supplied sort orders and selection criteria.

These structure-editing templates are being combined with a forms editor, which handles the element display functions. We are still working on other templates that would handle network or schematic displays, as shown in Figure 3. Such display forms are more difficult because of the layout and connection routing aids that should be provided automatically.

\section{Editing template system structure}

The editing template system itself consists of two processes. First, a new template is created using the template description editor. Second, instances of various templates are created. The relationship between the processes is shown in Figure 4.

The process begins with a template designer creating a description of a new template using the template description editor. The descriptions for all templates are stored in the template description file. A template description includes a slot for each piece of information that must be supplied when creating an instance of the template. The kinds of slots are STUF datatypes, fields from STUF objects, cursors, procedures, and constants. The slot description completely defines the nature of each piece of information. In addition to the slots that must be filled, the template description contains definitions for all service routines that the template will supply. Along with the template descriptions, a template designer also must write the code that actually implements the editing template and place the code in a library.

Using the instance class editor, an application programmer can define classes of template instances. For example, a template may exist for displaying alphabetized lists. From that template two instance classes might be created for displaying 
variable declarations and file names. The instance class for file names could then be used at runtime to create several different lists of files in separate windows. Each of these lists is an instance. Instance classes must be defined before compile time, since they depend on type declarations. Instances are created by the application at runtime using the generated instance class routines.

To aid the programmer in creating and using instance classes, the instance class editor uses the information in the template description file. The editor will also show the programmer all of the services that the template is making available, along with documentation about their use. This information comes from the template description file.

Because much of the information in an instance class is related to STUF files, the instance class editor also will load sample STUF files for the specific application and use the file's descriptive information to aid in class creation. From the instance class definitions, software is automatically generated, making the connections between the general-purpose template code and the specific application. All of this is then compiled and linked to form an interactive application.

\section{Summary}

Our user interface management system consists of a command-based dialogue editor called MIKE, which provides quick, easily learned prototyping of dialogues along with refinement of the dialogue via a profile editor. The interaction is viewed as an editing of a STUF format, which has the expressive power of both relational and linked data structures. Editing templates provide generic data editing and screen update procedures for various data display techniques.

The main difference between this approach and other UIMS approaches is that the interaction is viewed as a data-editing process rather than simply as an input dialogue-parsing problem or a screenmanagement problem. Having adopted this view, we can identify generic classes of display and editing techniques for various data organizations. Given such a class, a template is developed that carefully links the input dialogue with the display management functions to provide vastly improved interactive response. The class can then be applied to specific data-editing problems simply by binding the parameters. Assuming that we have an application whose data organization can suitably use the editing templates provided (which is an open-ended set), we can create an interactive browser/editor in a matter of days rather than months. For parts of an application not matching one of the existing templates, new code can be written and easily integrated with the templates. In fact, after such code has been written, it should be examined for its potential to become a new template itself.

\section{References}

1. J.J. Thomas and G. Hamlin, "Graphical Input Interaction Technique Workshop Summary," Computer Graphics, Vol. 17, No. 1, Jan. 1983, pp.5-16.

2. A. Kamran and M.B. Feldman, "Graphics Programming Independent of Interaction Techniques and Styles," Computer Graphics, Vol. 17, No. 1, Jan. 1983, pp.58-66.

3. M. Green, "The University of Alberta User Interface Management System," Computer Graphics (Proc. SIGGRAPH $85)$, Vol. 19, No. 3, July 1985, pp.205-213.

4. R.J.K. Jacob, "Using Formal Specifications in the Design of a Human-Computer Interface," Comm. ACM, Vol. 26, No. 4, Apr. 1983, pp.259-264.

5. J. van den Bos, M.J. Plasmeijer, and P.H. Hartel, "InputOutput Tools: A Language Facility for Interactive and RealTime Systems," IEEE Trans. Software Eng., Vol. 9, No. 3, May 1983, pp.247-259.

6. D.R. Olsen and E.P. Dempsey, "SYNGRAPH: A Graphical User Interface Generator," Computer Graphics (Proc. SIGGRAPH 83), Vol. 17, No. 3, July 1983, pp.43-50.

7. D.R. Olsen, E.P. Dempsey, and R.A. Rogge, "Input/Output Linkage in a User Interface Management System," Computer Graphics (Proc. SIGGRAPH 85), Vol. 19, No. 3, July 1985, pp.191-197.

8. D.R. Olsen, "Pushdown Automata for User Interface Management," ACM Trans. Graphics, Vol. 3, No. 3, July 1985 , pp.177-203.

9. D.R. Olsen, "User's Manual for MIKE-2.0," internal report, Comp. Sci. Dept., Brigham Young Univ., Provo, Utah, 1984.

10. M. Green, "Report on Dialogue Specification Tools," in User Interface Management Systems, G. Pfaff, ed., Springer-Verlag, Berlin, 1985, pp.9-20.

11. A. Goldberg and D. Robson, Smalltalk-80: The Language and Its Implementation, Addison-Wesley, Reading, Mass., 1983.

12. W. Buxton et al., "Toward a Comprehensive User Interface Management System," Computer Graphics (Proc. SIGGRAPH 83), Vol. 17, No. 3, July 1983, pp.35-42.

13. N. Gehani, Ada: An Advanced Introduction, Prentice-Hall, Englewood Cliffs, N.J., 1984.

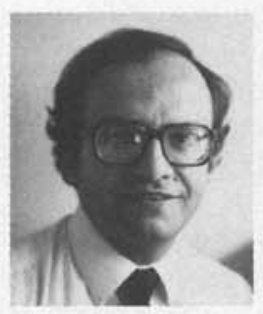

Dan R. Olsen, Jr., is an associate professor of computer science at Brigham Young University. From 1981 to 1985 he was on the computer science faculty at Arizona State University. He worked on the Burroughs Scientific Processor project at Burroughs Great Valley Labs from 1978 to 1980 . His research interests include support tools for user interface development, graphic programming systems, computationally active display structures, and compiler construction.

Olsen received the BS and MS in computer science from Brigham Young University and the $\mathrm{PhD}$ in computer and information science from the University of Pennsylvania. He is a member of the IEEE Computer Society and ACM.

Olsen can be contacted at the Computer Science Department, Brigham Young University, Provo, UT 84602. 